\title{
Search for Large Extra Spatial Dimensions in Dimuon Production with the Do Detector
}

V. M. Abazov, ${ }^{35}$ B. Abbott, ${ }^{72}$ M. Abolins, ${ }^{63}$ B. S. Acharya, ${ }^{29}$ M. Adams, ${ }^{50}$ T. Adams, ${ }^{48}$ M. Agelou, ${ }^{18}$ J.-L. Agram, ${ }^{19}$ S. H. Ahn, ${ }^{31}$ M. Ahsan, ${ }^{57}$ G. D. Alexeev,${ }^{35}$ G. Alkhazov, ${ }^{39}$ A. Alton, ${ }^{62}$ G. Alverson, ${ }^{61}$ G. A. Alves, ${ }^{2}$ M. Anastasoaie, ${ }^{34}$ T. Andeen, ${ }^{52}$ S. Anderson, ${ }^{44}$ B. Andrieu, ${ }^{17}$ Y. Arnoud,${ }^{14}$ A. Askew, ${ }^{48}$ B. Åsman, ${ }^{40}$ A. C. S. Assis Jesus,${ }^{3}$ O. Atramentov,${ }^{55}$ C. Autermann, ${ }^{21}$ C. Avila, ${ }^{8}$ F. Badaud,${ }^{13}$ A. Baden, ${ }^{59}$ L. Bagby, ${ }^{51}$ B. Baldin,${ }^{49}$ P. W. Balm,${ }^{33}$ P. Banerjee, ${ }^{29}$ S. Banerjee,${ }^{29}$ E. Barberis, ${ }^{61}$ P. Bargassa, ${ }^{76}$ P. Baringer, ${ }^{56}$ C. Barnes, ${ }^{42}$ J. Barreto, ${ }^{2}$ J. F. Bartlett,${ }^{49}$ U. Bassler, ${ }^{17}$ D. Bauer, ${ }^{53}$ A. Bean ${ }^{56}$ S. Beauceron, ${ }^{17}$ M. Begalli, ${ }^{3}$ M. Begel, ${ }^{68}$ A. Bellavance, ${ }^{65}$ S. B. Beri, ${ }^{27}$ G. Bernardi, ${ }^{17}$ R. Bernhard,${ }^{49, *}$ I. Bertram,${ }^{41, *}$ M. Besançon, ${ }^{18}$ R. Beuselinck, ${ }^{42}$ V. A. Bezzubov, ${ }^{38}$ P. C. Bhat, ${ }^{49}$ V. Bhatnagar, ${ }^{27}$ M. Binder, ${ }^{25}$ C. Biscarat,${ }^{41}$ K. M. Black, ${ }^{60}$ I. Blackler, ${ }^{42}$ G. Blazey, ${ }^{51}$ F. Blekman, ${ }^{42}$ S. Blessing, ${ }^{48}$ D. Bloch,${ }^{19}$ U. Blumenschein, ${ }^{23}$ A. Boehnlein, ${ }^{49}$ O. Boeriu, ${ }^{54}$ T. A. Bolton,${ }^{57}$ F. Borcherding, ${ }^{49}$ G. Borissov, ${ }^{41}$ K. Bos,${ }^{33}$ T. Bose,${ }^{67}$ A. Brandt,${ }^{74}$ R. Brock,${ }^{63}$ G. Brooijmans,${ }^{67}$ A. Bross ${ }^{49}$ N. J. Buchanan, ${ }^{48}$ D. Buchholz, ${ }^{52}$ M. Buehler, ${ }^{50}$ V. Buescher, ${ }^{23}$ S. Burdin, ${ }^{49}$ S. Burke, ${ }^{44}$ T. H. Burnett,${ }^{78}$ E. Busato, ${ }^{17}$ C. P. Buszello, ${ }^{42}$ J. M. Butler, ${ }^{60}$ J. Cammin, ${ }^{68}$ S. Caron, ${ }^{33}$ W. Carvalho, ${ }^{3}$ B. C. K. Casey, ${ }^{73}$ N. M. Cason,${ }^{54}$ H. Castilla-Valdez, ${ }^{32}$ S. Chakrabarti, ${ }^{29}$ D. Chakraborty, ${ }^{51}$ K. M. Chan ${ }^{68}$ A. Chandra, ${ }^{29}$ D. Chapin, ${ }^{73}$ F. Charles, ${ }^{19}$ E. Cheu, ${ }^{44}$ D. K. Cho, ${ }^{60}$ S. Choi ${ }^{47}$ B. Choudhary, ${ }^{28}$ T. Christiansen, ${ }^{25}$ L. Christofek, ${ }^{56}$ D. Claes,${ }^{65}$ B. Clément, ${ }^{19}$ C. Clément, ${ }^{40}$ Y. Coadou, ${ }^{5}$ M. Cooke,${ }^{76}$ W. E. Cooper ${ }^{49}$ D. Coppage,${ }^{56}$ M. Corcoran,${ }^{76}$ A. Cothenet, ${ }^{15}$ M.-C. Cousinou, ${ }^{15}$ B. Cox, ${ }^{43}$ S. Crépé-Renaudin, ${ }^{14}$ D. Cutts, ${ }^{73}$ H. da Motta, ${ }^{2}$ M. Das, ${ }^{58}$ B. Davies,${ }^{41}$ G. Davies,${ }^{42}$ G. A. Davis,${ }^{52}$ K. De,${ }^{74}$ P. de Jong, ${ }^{33}$ S. J. de Jong, ${ }^{34}$ E. De La Cruz-Burelo, ${ }^{62}$ C. De Oliveira Martins, ${ }^{3}$ S. Dean, ${ }^{43}$ J. D. Degenhardt, ${ }^{62}$ F. Déliot,${ }^{18}$ M. Demarteau, ${ }^{49}$ R. Demina,${ }^{68}$ P. Demine, ${ }^{18}$ D. Denisov, ${ }^{49}$ S. P. Denisov,${ }^{38}$ S. Desai, ${ }^{69}$ H. T. Diehl,${ }^{49}$ M. Diesburg, ${ }^{49}$ M. Doidge ${ }^{41}$ H. Dong, ${ }^{69}$ S. Doulas, ${ }^{61}$ L. V. Dudko, ${ }^{37}$ L. Duflot, ${ }^{16}$ S. R. Dugad, ${ }^{29}$ A. Duperrin, ${ }^{15}$ J. Dyer,${ }^{63}$ A. Dyshkant, ${ }^{51}$

M. Eads, ${ }^{51}$ D. Edmunds ${ }^{63}$ T. Edwards, ${ }^{43}$ J. Ellison, ${ }^{47}$ J. Elmsheuser, ${ }^{25}$ V. D. Elvira, ${ }^{49}$ S. Eno, ${ }^{59}$ P. Ermolov, ${ }^{37}$

O. V. Eroshin, ${ }^{38}$ J. Estrada, ${ }^{49}$ H. Evans, ${ }^{67}$ A. Evdokimov, ${ }^{36}$ V. N. Evdokimov, ${ }^{38}$ J. Fast,${ }^{49}$ S. N. Fatakia, ${ }^{60}$ L. Feligioni,${ }^{60}$ A. V. Ferapontov, ${ }^{38}$ T. Ferbel, ${ }^{68}$ F. Fiedler, ${ }^{25}$ F. Filthaut, ${ }^{34}$ W. Fisher, ${ }^{49}$ H. E. Fisk, ${ }^{49}$ I. Fleck, ${ }^{23}$ M. Fortner, ${ }^{51}$ H. Fox,${ }^{23}$ S. Fu, ${ }^{49}$ S. Fuess, ${ }^{49}$ T. Gadfort, ${ }^{78}$ C. F. Galea, ${ }^{34}$ E. Gallas, ${ }^{49}$ E. Galyaev,${ }^{54}$ C. Garcia, ${ }^{68}$ A. Garcia-Bellido, ${ }^{78}$ J. Gardner,${ }^{56}$ V. Gavrilov, ${ }^{36}$ A. Gay,${ }^{19}$ P. Gay, ${ }^{13}$ D. Gelé, ${ }^{19}$ R. Gelhaus, ${ }^{47}$ K. Genser, ${ }^{49}$ C. E. Gerber,${ }^{50}$ Y. Gershtein, ${ }^{48}$ D. Gillberg, ${ }^{5}$

G. Ginther, ${ }^{68}$ T. Golling, ${ }^{22}$ N. Gollub,${ }^{40}$ B. Gómez, ${ }^{8}$ K. Gounder ${ }^{49}$ A. Goussiou ${ }^{54}$ P. D. Grannis ${ }^{69}$ S. Greder, ${ }^{3}$

H. Greenlee, ${ }^{49}$ Z. D. Greenwood, ${ }^{58}$ E. M. Gregores, ${ }^{4}$ Ph. Gris, ${ }^{13}$ J.-F. Grivaz, ${ }^{16}$ L. Groer, ${ }^{67}$ S. Grünendahl,${ }^{49}$ M. W. Grünewald ${ }^{30}$ S. N. Gurzhiev, ${ }^{38}$ G. Gutierrez, ${ }^{49}$ P. Gutierrez,${ }^{72}$ A. Haas, ${ }^{67}$ N. J. Hadley, ${ }^{59}$ S. Hagopian, ${ }^{48}$ I. Hall, ${ }^{72}$ R. E. Hall, ${ }^{46}$ C. Han, ${ }^{62}$ L. Han,${ }^{7}$ K. Hanagaki, ${ }^{49}$ K. Harder, ${ }^{57}$ A. Harel, ${ }^{26}$ R. Harrington, ${ }^{61}$ J. M. Hauptman,${ }^{55}$ R. Hauser,${ }^{63}$ J. Hays,${ }^{52}$ T. Hebbeker, ${ }^{21}$ D. Hedin, ${ }^{51}$ J. M. Heinmiller, ${ }^{50}$ A. P. Heinson, ${ }^{47}$ U. Heintz, ${ }^{60}$ C. Hensel,${ }^{56}$ G. Hesketh, ${ }^{61}$ M. D. Hildreth, ${ }^{54}$ R. Hirosky, ${ }^{77}$ J. D. Hobbs, ${ }^{69}$ B. Hoeneisen, ${ }^{12}$ M. Hohlfeld ${ }^{24}$ S. J. Hong, ${ }^{31}$ R. Hooper, ${ }^{73}$ P. Houben, ${ }^{33}$ Y. Hu, ${ }^{69}$ J. Huang, ${ }^{53}$ V. Hynek, ${ }^{9}$ I. Iashvili, ${ }^{47}$ R. Illingworth, ${ }^{49}$ A. S. Ito, ${ }^{49}$ S. Jabeen, ${ }^{56}$ M. Jaffré, ${ }^{16}$ S. Jain, ${ }^{72}$ V. Jain, ${ }^{70}$ K. Jakobs,${ }^{23}$ A. Jenkins, ${ }^{42}$ R. Jesik, ${ }^{42}$ K. Johns, ${ }^{44}$ M. Johnson, ${ }^{49}$ A. Jonckheere, ${ }^{49}$ P. Jonsson, ${ }^{42}$ A. Juste, ${ }^{49}$ D. Käfer, ${ }^{21}$

S. Kahn, ${ }^{70}$ E. Kajfasz, ${ }^{15}$ A. M. Kalinin, ${ }^{35}$ J. Kalk, ${ }^{63}$ D. Karmanov, ${ }^{37}$ J. Kasper, ${ }^{60}$ D. Kau, ${ }^{48}$ R. Kaur, ${ }^{27}$ R. Kehoe, ${ }^{75}$ S. Kermiche, ${ }^{15}$ S. Kesisoglou, ${ }^{73}$ A. Khanov, ${ }^{68}$ A. Kharchilava, ${ }^{54}$ Y. M. Kharzheev, ${ }^{35}$ H. Kim, ${ }^{74}$ T. J. Kim, ${ }^{31}$ B. Klima, ${ }^{49}$ J. M. Kohli, ${ }^{27}$ J.-P. Konrath, ${ }^{23}$ M. Kopal, ${ }^{72}$ V. M. Korablev, ${ }^{38}$ J. Kotcher, ${ }^{70}$ B. Kothari, ${ }^{67}$ A. Koubarovsky, ${ }^{37}$ A. V. Kozelov, ${ }^{38}$ J. Kozminski, ${ }^{63}$ A. Kryemadhi, ${ }^{77}$ S. Krzywdzinski, ${ }^{49}$ Y. Kulik, ${ }^{49}$ A. Kumar ${ }^{28}$ S. Kunori ${ }^{59}$ A. Kupco, ${ }^{11}$ T. Kurča, ${ }^{20}$ J. Kvita, ${ }^{9}$ S. Lager, ${ }^{40}$ N. Lahrichi, ${ }^{18}$ G. Landsberg, ${ }^{73}$ J. Lazoflores, ${ }^{48}$ A.-C. Le Bihan, ${ }^{19}$ P. Lebrun, ${ }^{20}$ W. M. Lee, ${ }^{48}$ A. Leflat,${ }^{37}$ F. Lehner, ${ }^{49, *}$ C. Leonidopoulos, ${ }^{67}$ J. Leveque ${ }^{44}$ P. Lewis $,{ }^{42} \mathrm{~J} . \mathrm{Li},{ }^{74}$ Q. Z. Li,${ }^{49}$ J. G. R. Lima,${ }^{51}$ D. Lincoln,${ }^{49}$ S. L. Linn, ${ }^{48}$ J. Linnemann, ${ }^{63}$ V. V. Lipaev, ${ }^{38}$ R. Lipton, ${ }^{49}$ L. Lobo, ${ }^{42}$ A. Lobodenko, ${ }^{39}$ M. Lokajicek, ${ }^{11}$ A. Lounis, ${ }^{19}$ P. Love, ${ }^{41}$ H. J. Lubatti, ${ }^{78}$ L. Lueking, ${ }^{49}$ M. Lynker, ${ }^{54}$ A. L. Lyon, ${ }^{49}$ A. K. A. Maciel,,${ }^{51}$ R. J. Madaras, ${ }^{45}$ P. Mättig, ${ }^{26}$

C. Magass, ${ }^{21}$ A. Magerkurth, ${ }^{62}$ A.-M. Magnan, ${ }^{14}$ N. Makovec,${ }^{16}$ P. K. Mal, ${ }^{29}$ H. B. Malbouisson, ${ }^{3}$ S. Malik, ${ }^{65}$

V. L. Malyshev, ${ }^{35}$ H. S. Mao, ${ }^{6}$ Y. Maravin, ${ }^{49}$ M. Martens, ${ }^{49}$ S.E. K. Mattingly, ${ }^{73}$ A. A. Mayorov, ${ }^{38}$ R. McCarthy, ${ }^{69}$ R. McCroskey, ${ }^{44}$ D. Meder, ${ }^{24}$ A. Melnitchouk, ${ }^{64}$ A. Mendes, ${ }^{15}$ M. Merkin, ${ }^{37}$ K. W. Merritt, ${ }^{49}$ A. Meyer, ${ }^{21}$ J. Meyer, ${ }^{22}$ M. Michaut, ${ }^{18}$ H. Miettinen, ${ }^{76}$ J. Mitrevski, ${ }^{67}$ J. Molina, ${ }^{3}$ N. K. Mondal, ${ }^{29}$ R. W. Moore, ${ }^{5}$ T. Moulik, ${ }^{56}$ G. S. Muanza, ${ }^{20}$ M. Mulders, ${ }^{49}$ L. Mundim, ${ }^{3}$ Y. D. Mutaf, ${ }^{69}$ E. Nagy, ${ }^{15}$ M. Narain, ${ }^{60}$ N. A. Naumann, ${ }^{34}$ H. A. Neal, ${ }^{62}$ J.P. Negret, ${ }^{8}$ S. Nelson, ${ }^{48}$ P. Neustroev,${ }^{39}$ C. Noeding,${ }^{23}$ A. Nomerotski,${ }^{49}$ S. F. Novaes, ${ }^{4}$ T. Nunnemann,${ }^{25}$ E. Nurse,${ }^{43}$ V. O’Dell ${ }^{49}$ D. C. O'Neil,${ }^{5}$ V. Oguri, ${ }^{3}$ N. Oliveira,${ }^{3}$ N. Oshima,${ }^{49}$ G. J. Otero y Garzón,${ }^{50}$ P. Padley, ${ }^{76}$ N. Parashar,${ }^{58}$ S. K. Park, ${ }^{31}$ J. Parsons, ${ }^{67}$ R. Partridge, ${ }^{73}$ N. Parua,${ }^{69}$ A. Patwa,${ }^{70}$ G. Pawloski, ${ }^{76}$ P. M. Perea,${ }^{47}$ E. Perez ${ }^{18}$ P. Pétroff, ${ }^{16}$ M. Petteni, ${ }^{42}$ R. Piegaia, ${ }^{1}$ M.-A. Pleier,${ }^{68}$ P. L. M. Podesta-Lerma, ${ }^{32}$ V. M. Podstavkov, ${ }^{49}$ Y. Pogorelov, ${ }^{54}$ M.-E. Pol, ${ }^{2}$ A. Pompoš,${ }^{72}$ 
B. G. Pope ${ }^{63}$ W. L. Prado da Silva, ${ }^{3}$ H. B. Prosper, ${ }^{48}$ S. Protopopescu, ${ }^{70}$ J. Qian, ${ }^{62}$ A. Quadt, ${ }^{22}$ B. Quinn, ${ }^{64}$ K. J. Rani, ${ }^{29}$ K. Ranjan, ${ }^{28}$ P. A. Rapidis, ${ }^{49}$ P. N. Ratoff, ${ }^{41}$ S. Reucroft,${ }^{61}$ M. Rijssenbeek,${ }^{69}$ I. Ripp-Baudot, ${ }^{19}$ F. Rizatdinova,${ }^{57}$ S. Robinson, ${ }^{42}$ R. F. Rodrigues, ${ }^{3}$ C. Royon, ${ }^{18}$ P. Rubinov, ${ }^{49}$ R. Ruchti,${ }^{54}$ V. I. Rud ${ }^{37}$ G. Sajot, ${ }^{14}$ A. Sánchez-Hernández, ${ }^{32}$ M. P. Sanders ${ }^{59}$ A. Santoro, ${ }^{3}$ G. Savage, ${ }^{49}$ L. Sawyer, ${ }^{58}$ T. Scanlon, ${ }^{42}$ D. Schaile, ${ }^{25}$ R. D. Schamberger, ${ }^{69}$ Y. Scheglov, ${ }^{39}$ H. Schellman, ${ }^{52}$ P. Schieferdecker, ${ }^{25}$ C. Schmitt, ${ }^{26}$ C. Schwanenberger, ${ }^{22}$ A. Schwartzman, ${ }^{66}$ R. Schwienhorst,${ }^{63}$ S. Sengupta, ${ }^{48}$ H. Severini, ${ }^{72}$ E. Shabalina, ${ }^{50}$ M. Shamim, ${ }^{57}$ V. Shary, ${ }^{18}$ A. A. Shchukin, ${ }^{38}$ W. D. Shephard, ${ }^{54}$ R. K. Shivpuri, ${ }^{28}$ D. Shpakov, ${ }^{61}$ R. A. Sidwell, ${ }^{57}$ V. Simak, ${ }^{10}$ V. Sirotenko, ${ }^{49}$ P. Skubic, ${ }^{72}$ P. Slattery, ${ }^{68}$ R. P. Smith, ${ }^{49}$ K. Smolek, ${ }^{10}$ G. R. Snow, ${ }^{65}$ J. Snow, ${ }^{71}$ S. Snyder,${ }^{70}$ S. Söldner-Rembold, ${ }^{43}$ X. Song, ${ }^{51}$ L. Sonnenschein, ${ }^{17}$ A. Sopczak,${ }^{41}$ M. Sosebee, ${ }^{74}$ K. Soustruznik, ${ }^{9}$ M. Souza, ${ }^{2}$ B. Spurlock, ${ }^{74}$ N. R. Stanton, ${ }^{57}$ J. Stark, ${ }^{14}$ J. Steele, ${ }^{58}$ K. Stevenson, ${ }^{53}$ V. Stolin, ${ }^{36}$ A. Stone,${ }^{50}$ D. A. Stoyanova, ${ }^{38}$ J. Strandberg, ${ }^{40}$ M. A. Strang,${ }^{74}$ M. Strauss, ${ }^{72}$ R. Ströhmer, ${ }^{25}$ D. Strom, ${ }^{52}$ M. Strovink ${ }^{45}$ L. Stutte ${ }^{49}$ S. Sumowidagdo, ${ }^{48}$ A. Sznajder, ${ }^{3}$ M. Talby, ${ }^{15}$ P. Tamburello, ${ }^{44}$ W. Taylor, ${ }^{5}$ P. Telford, ${ }^{43}$ J. Temple, ${ }^{44}$ M. Titov,${ }^{23}$ M. Tomoto, ${ }^{49}$ T. Toole,${ }^{59}$ J. Torborg,${ }^{54}$ S. Towers,${ }^{69}$ T. Trefzger ${ }^{24}$ S. Trincaz-Duvoid, ${ }^{17}$ D. Tsybychev, ${ }^{69}$ B. Tuchming, ${ }^{18}$ C. Tully, ${ }^{66}$ A. S. Turcot ${ }^{43}$ P. M. Tuts, ${ }^{67}$ L. Uvarov, ${ }^{39}$ S. Uvarov, ${ }^{39}$ S. Uzunyan, ${ }^{51}$ B. Vachon, ${ }^{5}$ P. J. van den Berg, ${ }^{33}$ R. Van Kooten, ${ }^{53}$ W. M. van Leeuwen,${ }^{33}$ N. Varelas, ${ }^{50}$ E. W. Varnes, ${ }^{44}$ A. Vartapetian, ${ }^{74}$ I. A. Vasilyev, ${ }^{38}$ M. Vaupel, ${ }^{26}$ P. Verdier, ${ }^{20}$ L. S. Vertogradov, ${ }^{35}$ M. Verzocchi,${ }^{59}$ F. Villeneuve-Seguier,${ }^{42}$ J.-R. Vlimant, ${ }^{17}$ E. Von Toerne, ${ }^{57}$ M. Vreeswijk, ${ }^{33}$ T. Vu Anh,${ }^{16}$ H. D. Wahl, ${ }^{48}$ L. Wang, ${ }^{59}$ J. Warchol, ${ }^{54}$ G. Watts, ${ }^{78}$ M. Wayne, ${ }^{54}$ M. Weber, ${ }^{49}$ H. Weerts, ${ }^{63} \mathrm{~N}$. Wermes,${ }^{22} \mathrm{M}$. Wetstein, ${ }^{59}$ A. White, ${ }^{74} \mathrm{~V}$. White,${ }^{49}$ D. Wicke, ${ }^{49}$ D. A. Wijngaarden,${ }^{34}$ G. W. Wilson, ${ }^{56}$ S. J. Wimpenny, ${ }^{47}$ J. Wittlin, ${ }^{60}$ M. Wobisch, ${ }^{49}$ J. Womersley, ${ }^{49}$ D. R. Wood, ${ }^{61}$ T. R. Wyatt, ${ }^{43}$ Q. Xu, ${ }^{62}$ N. Xuan, ${ }^{54}$ S. Yacoob, ${ }^{52}$ R. Yamada, ${ }^{49}$ M. Yan, ${ }^{59}$ T. Yasuda, ${ }^{49}$ Y. A. Yatsunenko, ${ }^{35}$ Y. Yen, ${ }^{26}$ K. Yip, ${ }^{70}$ H. D. Yoo, ${ }^{73}$ S. W. Youn, ${ }^{52}$ J. Yu, ${ }^{74}$ A. Yurkewicz, ${ }^{69}$ A. Zabi, ${ }^{16}$ A. Zatserklyaniy, ${ }^{51}$ M. Zdrazil,${ }^{69}$ C. Zeitnitz, ${ }^{24}$ D. Zhang, ${ }^{49}$ X. Zhang, ${ }^{72}$ T. Zhao, ${ }^{78}$ Z. Zhao, ${ }^{62}$ B. Zhou, ${ }^{62}$ J. Zhu, ${ }^{69}$ M. Zielinski, ${ }^{68}$ D. Zieminska, ${ }^{53}$ A. Zieminski, ${ }^{53}$ R. Zitoun, ${ }^{69}$ V. Zutshi, ${ }^{51}$ and E. G. Zverev ${ }^{37}$

\title{
(D0 Collaboration)
}

\author{
${ }^{1}$ Universidad de Buenos Aires, Buenos Aires, Argentina \\ ${ }^{2}$ LAFEX, Centro Brasileiro de Pesquisas Físicas, Rio de Janeiro, Brazil \\ ${ }^{3}$ Universidade do Estado do Rio de Janeiro, Rio de Janeiro, Brazil \\ ${ }^{4}$ Instituto de Física Teórica, Universidade Estadual Paulista, São Paulo, Brazil \\ ${ }^{5}$ University of Alberta, Edmonton, Alberta, Canada, Simon Fraser University, Burnaby, British Columbia, Canada, York University, \\ Toronto, Ontario, Canada, and McGill University, Montreal, Quebec, Canada \\ ${ }^{6}$ Institute of High Energy Physics, Beijing, People's Republic of China \\ ${ }^{7}$ University of Science and Technology of China, Hefei, People's Republic of China \\ ${ }^{8}$ Universidad de los Andes, Bogotá, Colombia \\ ${ }^{9}$ Center for Particle Physics, Charles University, Prague, Czech Republic \\ ${ }^{10}$ Czech Technical University, Prague, Czech Republic \\ ${ }^{11}$ Center for Particle Physics, Institute of Physics, Academy of Sciences of the Czech Republic, Prague, Czech Republic \\ ${ }^{12}$ Universidad San Francisco de Quito, Quito, Ecuador \\ ${ }^{13}$ Laboratoire de Physique Corpusculaire, IN2P3-CNRS, Université Blaise Pascal, Clermont-Ferrand, France \\ ${ }^{14}$ Laboratoire de Physique Subatomique et de Cosmologie, IN2P3-CNRS, Universite de Grenoble 1, Grenoble, France \\ ${ }^{15}$ CPPM, IN2P3-CNRS, Université de la Méditerranée, Marseille, France \\ ${ }^{16}$ IN2P3-CNRS, Laboratoire de l'Accélérateur Linéaire, Orsay, France \\ ${ }^{17}$ LPNHE, IN2P3-CNRS, Universités Paris VI and VII, Paris, France \\ ${ }^{18}$ DAPNIA/Service de Physique des Particules, CEA, Saclay, France \\ ${ }^{19}$ IReS, IN2P3-CNRS, Université Louis Pasteur, Strasbourg, France, and Université de Haute Alsace, Mulhouse, France \\ ${ }^{20}$ Institut de Physique Nucléaire de Lyon, IN2P3-CNRS, Université Claude Bernard, Villeurbanne, France \\ ${ }^{21}$ III. Physikalisches Institut A, RWTH Aachen, Aachen, Germany \\ ${ }^{22}$ Physikalisches Institut, Universität Bonn, Bonn, Germany \\ ${ }^{23}$ Physikalisches Institut, Universität Freiburg, Freiburg, Germany \\ ${ }^{24}$ Institut für Physik, Universität Mainz, Mainz, Germany \\ ${ }^{25}$ Ludwig-Maximilians-Universität München, München, Germany \\ ${ }^{26}$ Fachbereich Physik, University of Wuppertal, Wuppertal, Germany \\ ${ }^{27}$ Panjab University, Chandigarh, India \\ ${ }^{28}$ Delhi University, Delhi, India \\ ${ }^{29}$ Tata Institute of Fundamental Research, Mumbai, India \\ ${ }^{30}$ University College Dublin, Dublin, Ireland
}




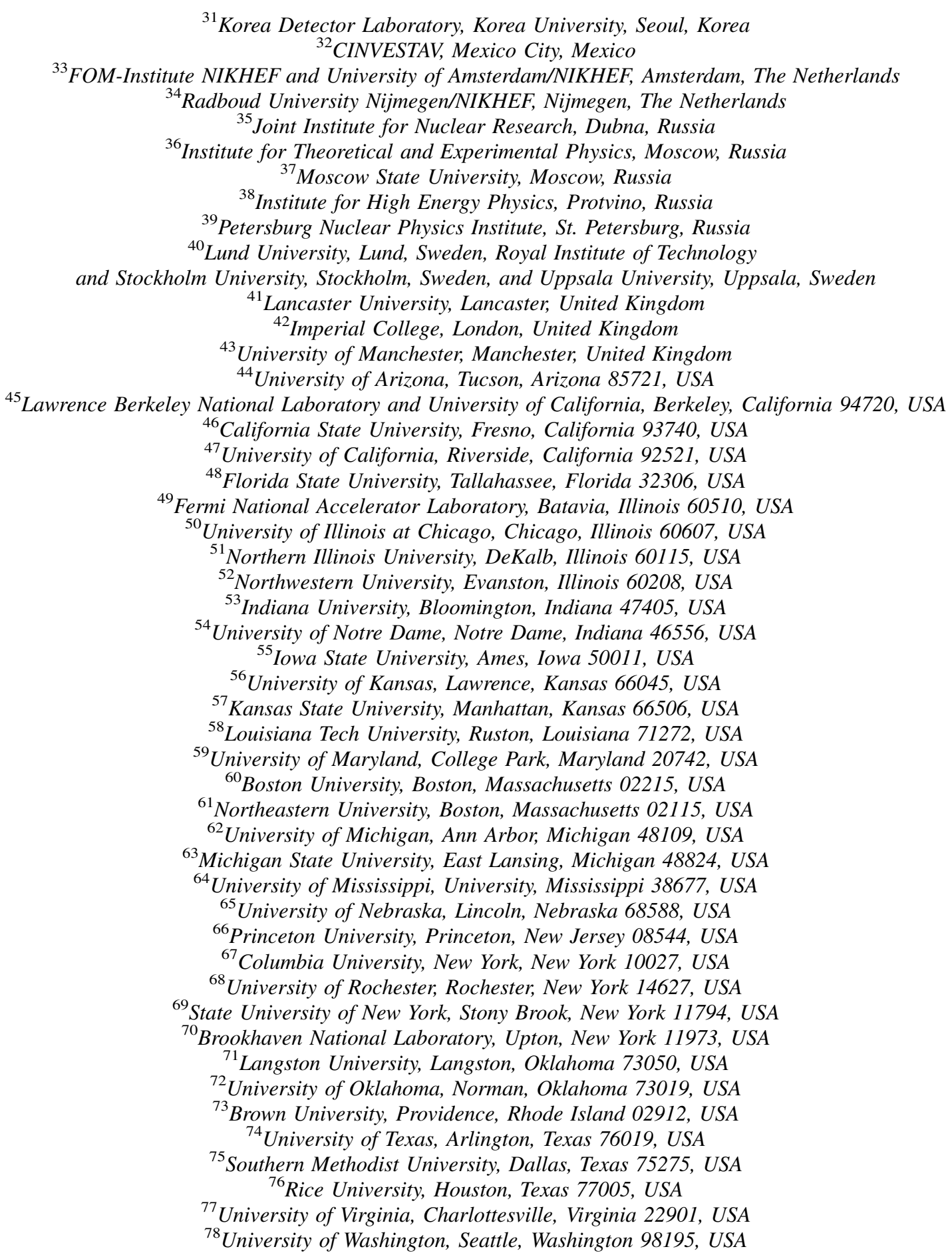
(Received 27 June 2005; published 13 October 2005)

We present the results of a search for the effects of large extra spatial dimensions in $p \bar{p}$ collisions at $\sqrt{s}=1.96 \mathrm{TeV}$ in events containing a pair of energetic muons. The data correspond to $246 \mathrm{pb}^{-1} \mathrm{of}^{-}$ integrated luminosity collected by the D0 experiment at the Fermilab Tevatron Collider. Good agreement with the expected background was found, yielding no evidence for large extra dimensions. We set $95 \%$ C.L. lower limits on the fundamental Planck scale between 0.85 and $1.27 \mathrm{TeV}$ within several formalisms. These are the most stringent limits achieved in the dimuon channel to date. 
In their 1998 paper, Arkani-Hamed, Dimopoulos, and Dvali (ADD) suggested that the seemingly unreachable Planck energy scale (conventionally thought to be $M_{\mathrm{Pl}} \sim$ $10^{19} \mathrm{GeV}$ ) may be, in fact, much lower, i.e., within the reach of current and planned future colliders [1]. They postulated that the standard model (SM) particles and gauge interactions are confined to a three-dimensional "brane" embedded in a "multiverse," which consists of the three standard plus $n$ additional compact spatial dimensions. However, gravitons in this framework can propagate in the entire multiverse. The gravitons propagating in compact extra dimensions appear as a tower of KaluzaKlein (KK) excited modes from the point of view of the $\mathrm{SM}$ brane. Furthermore, the radius of compactification $(R)$ of extra dimensions in the ADD model is much larger than either the Planck or electroweak length, and may be as large as $\sim 1 \mathrm{~mm}$. Since gravitons are free to propagate in these large extra dimensions, the gravitational interaction would appear suppressed on the SM brane, due to the extra volume gravity permeates. Consequently, while the apparent Planck scale is $\sim 10^{19} \mathrm{GeV}$, with respect to the $(3+n)$-dimensional space, the fundamental Planck scale $\left(M_{S}\right)$ can be as low as $\sim 1 \mathrm{TeV}$, thus eliminating the hierarchy problem of the SM.

The phenomenological consequences of the ADD model have been a subject of intense study in recent years. For a review of the possible effects of large extra dimensions, ranging from modification of Newtonian gravity to black hole production at future colliders, see, e.g., Refs. [2,3].

In this Letter, we describe a search for the effects of large extra dimensions via virtual Kaluza-Klein graviton $\left(G_{\mathrm{KK}}\right)$ exchange in $p \bar{p}$ collisions resulting in the dimuon final state. Technically, virtual graviton effects are sensitive to the ultraviolet cutoff required to keep the divergent sum over the KK states finite [4-6], rather than the fundamental Planck scale. As the two scales are expected to be closely related, we do not distinguish between them in this analysis. The search is based on $246 \pm 16 \mathrm{pb}^{-1}$ of data collected in 2002-2004 by the D0 detector operating at the Fermilab Tevatron Collider at $\sqrt{s}=1.96 \mathrm{TeV}$. We used the method of Refs. [7,8], in which the dilepton invariant mass $(M)$ and the cosine of the scattering angle $\left(\cos \theta^{*}\right)$ in the dilepton center of mass frame are analyzed simultaneously for the effects of large extra dimensions. This is the first search for large extra dimensions in the dimuon channel at a hadron collider. Previous searches for virtual graviton effects in various channels at LEP, HERA, and the Tevatron are reviewed in detail in Ref. [3].

The dimuon production cross section in the presence of extra dimensions is given by [4-6]

$$
\frac{d^{2} \sigma}{d M d \cos \theta^{*}}=f_{\mathrm{SM}}+f_{\mathrm{int}} \eta_{G}+f_{\mathrm{KK}} \eta_{G}^{2},
$$

where $f_{\mathrm{SM}}, f_{\text {int }}$, and $f_{\mathrm{KK}}$ are functions of $\left(M, \cos \theta^{*}\right)$ and denote the SM, interference, and $G_{\mathrm{KK}}$ terms. The effects of large extra dimensions are parametrized via a single variable $\eta_{G}=\mathcal{F} / M_{S}^{4}$, where $\mathcal{F}$ is a dimensionless parameter of order unity. Three different formalisms for $\mathcal{F}$ are explored in this analysis:

$$
\mathcal{F}=1
$$

[Giudice-Rattazzi-Wells (GRW) [4] ];

$$
\mathcal{F}= \begin{cases}\log \left(\frac{M_{S}^{2}}{M^{2}}\right), & n=2 \\ \frac{2}{n-2}, & n>2\end{cases}
$$

[Han-Lykken-Zhang (HLZ) [5] ];

$$
\mathcal{F}=\frac{2 \lambda}{\pi}= \pm \frac{2}{\pi}
$$

(Hewett [6]). In Eq. (4) $\lambda= \pm 1$ indicates whether virtual graviton exchange interferes constructively or destructively with SM processes. While virtual graviton exchange does not depend strongly on $n$ (the number of extra dimensions), the HLZ formalism for $\mathcal{F}$ does explore this dependence explicitly.

The D0 detector and its data acquisition system are described in detail elsewhere [9]. Here we give a brief description of the components used in the analysis. At the center of the D0 detector is the central-tracking system, which consists of a silicon microstrip tracker and a central fiber tracker. Both trackers are located within a $2 \mathrm{~T}$ axial magnetic field [9], with designs optimized for tracking and vertexing at pseudorapidities $\left|\eta_{d}\right|<3$. The pseudorapidity $\eta$ is defined as $-\ln \left(\tan \frac{\theta}{2}\right)$, where $\theta$ is the polar angle with respect to the proton-beam direction, as measured from the interaction vertex. We also define $\eta_{d}$, which is the pseudorapidity based on the polar angle measured from the geometric center of the detector. The nearly hermetic uranium/liquid-argon calorimeter is used to measure energies of electrons, photons, and hadrons [10]. The muon system covers $\left|\eta_{d}\right|<2$ and consists of a layer of tracking detectors and scintillation trigger counters in front of $1.8 \mathrm{~T}$ iron toroidal magnets, followed by two more similar layers of detectors outside the toroids [11]. Luminosity is measured using plastic scintillator arrays placed in the large $\left|\eta_{d}\right|$ (forward) regions of the detector.

The sample of candidate events used in the search was collected with a set of triggers that require either one or two muon candidates in the muon system. After detailed event reconstruction, an event must contain at least two muon candidates, each matched with a track in the central tracker, which is used for muon momentum measurement. The matching tracks were required to have transverse momenta $p_{T}>15 \mathrm{GeV}$, at least one hit in the silicon microstrip tracker, and at least nine hits in the central fiber tracker. The latter two requirements ensure reliable momentum measurement, especially at high $p_{T}$. To reduce 
background from cosmic rays, we introduced additional criteria. Since cosmic muons are not correlated with the beam crossing, we required the muon arrival time, as measured in the muon system scintillation counters, to be within $10 \mathrm{~ns}$ (4 standard deviations) of the expected arrival time for a highly relativistic particle produced in a $p \bar{p}$ collision in the center of the detector [11]. Furthermore, dimuon events that originate from a cosmic muon are back to back in $\eta$. This is because the same cosmic muon is reconstructed twice in the event, once when entering the detector and once when leaving it. Consequently, dimuon events from cosmic rays should have $\eta_{1}+\eta_{2} \approx 0$. In true dimuon events originating from $p \bar{p}$ collisions, the two muons are generally not back to back in $\eta$ due to a longitudinal boost of the dimuon system. Therefore the sum $\eta_{1}+\eta_{2}$ was required to be away from zero by at least 5 standard deviations of the Gaussian distribution as observed in a cosmic ray sample. The signal efficiency for this selection is $(99 \pm 1) \%$. After the above selections the cosmic muon contamination in the candidate sample is negligible.

Muons from graviton decay are expected to be isolated from other energetic particles or jets. In contrast, high $p_{T}$ muons from $b$ and $c$ quark decays tend to be nonisolated. To reduce this copious background, we required that each muon have the following: (i) $\sum_{\mathcal{R}=0.5}\left(p_{T}\right)<2.5 \mathrm{GeV}$, where $\sum_{\mathcal{R}=0.5}\left(p_{T}\right)$ is the scalar sum of the $p_{T}$ of all additional tracks contained within a cone of radius $\mathcal{R}=$ $\sqrt{(\Delta \eta)^{2}+(\bar{\phi} \phi)^{2}}=0.5$ [12] centered on the muon track; and (ii) $\sum_{\mathcal{R}=0.4}\left(E_{T}\right)-\sum_{\mathcal{R}=0.1}\left(E_{T}\right)<2.5 \mathrm{GeV}$, where the $E_{T}$ 's are the transverse energies of the calorimeter cells within the respective cones centered on the muon track.

After all the selections are applied, the efficiency per muon is $(80 \pm 4) \%$, as measured with $Z \rightarrow \mu \mu$ events. This includes the efficiency for muon and track reconstruction, track matching, number of tracker hits, and cosmic ray muon vetoes, as well as the isolation selections.

Because this analysis focuses on very high- $p_{T}$ objects, the most problematic background is that from mismeasured Drell-Yan (DY) events that appear to have very high mass. This occurs because if a muon's $p_{T}$ is mismeasured toward a higher value it tends to be grossly mismeasured high, and thus the reconstructed mass also tends to be much higher than it actually is. This is due to the resolution of the central tracker, which is approximately Gaussian in $1 / p_{T}$ with a typical $\sigma=0.00272$. We reduce the effect by scaling the $p_{T}$ of each muon track to a weighted average based on the original track $\frac{1}{p_{T}}$ measurement and its uncertainty. For example, in the highest-mass event the original muon $p_{T}$ 's were 250 and $1000 \mathrm{GeV}$, while after $p_{T}$ fixing both $p_{T}$ 's became $400 \mathrm{GeV}$. This procedure assumes the two muons' transverse momenta should be equal. For high mass objects such as a KK graviton the equal momenta assumption would be accurate. The following equation illustrates the procedure:

$$
\frac{1}{p_{T_{1}}^{\prime}}=\frac{1}{p_{T_{2}}^{\prime}}=\frac{\left|w_{1} / p_{T_{1}}+\epsilon w_{2} / p_{T_{2}}\right|}{w_{1}+w_{2}}
$$

where $p_{T_{1,2}}$ are the original $p_{T}$ 's of the two muons, $w_{1,2}=$ $1 / \sigma^{2}\left(1 / p_{T_{1,2}}\right)$ are the Gaussian weights, and $\sigma\left(1 / p_{T_{1,2}}\right)$ is the uncertainty on $1 / p_{T_{1,2}}$ measured from the shape of the $Z$ boson peak. In the equation above $\epsilon=+1$ for muon pairs with opposite charge and $\epsilon=-1$ for same charge muon pairs. This search does not require that the two muons have opposite charges, because the efficiency of such a requirement degrades quickly at high masses and the requirement does not reduce the already low background there.

Based on the values of $1 / p_{T}^{\prime}$ and the original angular information, a new momentum was calculated for the two muons in the event. The same procedure was also applied to the simulated signal and background. After the reweighting was done, we required the dimuon mass $M$ to be greater than $50 \mathrm{GeV}$, which resulted in a final candidate sample of 17128 events, most of them in the vicinity of the $Z$ boson peak.

We modeled the effects of large extra dimensions via the parton-level leading-order (LO) Monte Carlo (MC) generator of Ref. [7], augmented with a parametric simulation of the D0 detector. The simulation takes into account detector acceptance and resolution for muons. The generator includes effects of initial state radiation as described below, and different parton distribution functions (PDF). We used the leading-order CTEQ5L [13] PDF to estimate the nominal prediction. The parameters of the detector model were tuned using $Z \rightarrow \mu \mu$ events. The simulation includes SM DY contributions $\left(Z / \gamma^{*}\right)$, Kaluza-Klein graviton exchange diagrams, and their interference.

Since the MC generator contains only the LO partonlevel processes, we modeled next-to-leading-order (NLO) effects by adding a transverse momentum to the dimuon system. The model is based on the transverse momentum spectrum of dielectron candidates observed in the calorimeter, which provides better high energy resolution capability than that of the central-tracking system. Since the parton-level cross section is calculated at LO, we accounted for the NLO enhancement in the SM background by scaling the cross sections by a constant $K$ factor of 1.34 [14]. We assigned $a \pm 5 \%$ systematic uncertainty on the value of the $K$ factor to account for its mass dependence. We assumed the same constant $K$ factor for the extra dimensions signal. Recent NLO calculations of the virtual graviton exchange cross section [15] showed that such a choice of signal $K$ factor is a reasonable assumption. That study calculated the NLO $K$ factor to be 1.3 for masses around $500 \mathrm{GeV}$.

The main SM source of isolated dimuons is DY production, which was modeled via the MC discussed above. Other SM sources (such as $b \bar{b}, Z \gamma, W W, Z \rightarrow \tau \tau$, and $t \bar{t}$ production) are negligible, as they either have small cross sections compared to that for DY or are eliminated by the 

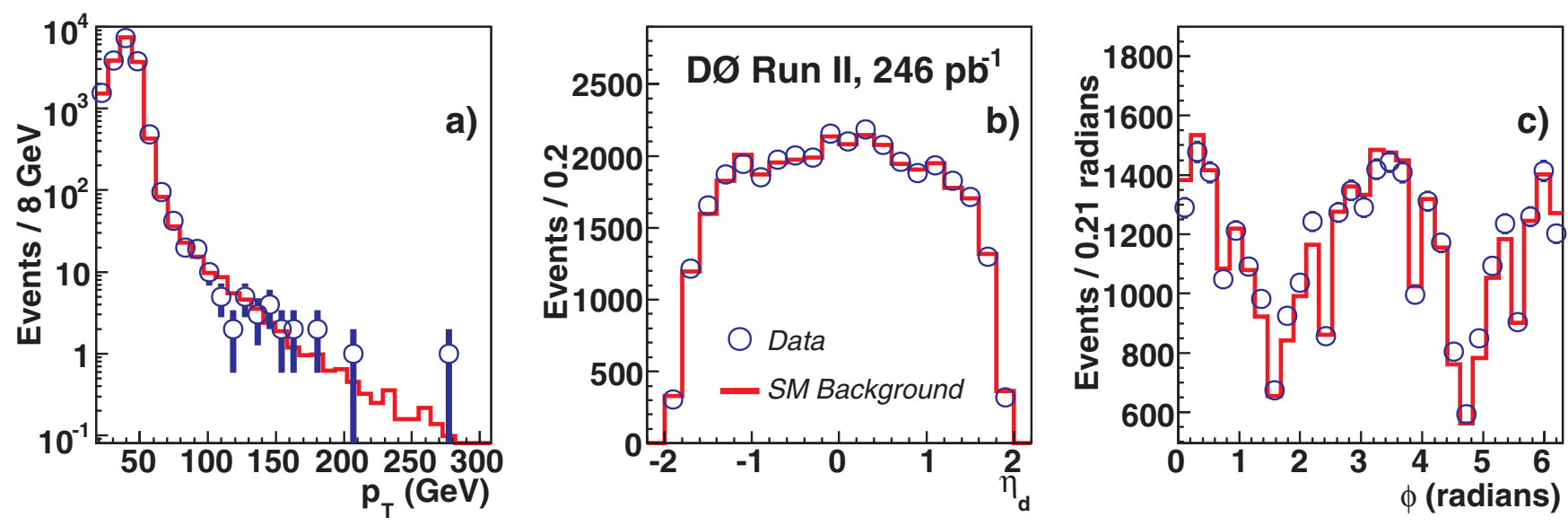

FIG. 1 (color online). Comparison of data (circles with error bars) and SM predictions (histogram) in $p_{T}, \eta_{d}$, and $\phi[12]$ of the muons in the event. The dips in the azimuthal angle reflect detector acceptance.

event selection. The SM DY background prediction reproduces the main kinematic characteristics of the candidate sample, as illustrated in Fig. 1.

Figure 2 shows the two-dimensional distribution in $M$ vs $\left|\cos \theta^{*}\right|$ for the SM background (other backgrounds are negligible and ignored), the sum of the background and an extra dimensions signal of $\eta_{G}=3 \mathrm{TeV}^{-4}$, and the data. The data agree with the background prediction and do not exhibit evidence for large extra dimensions, which would produce an excess of events at high mass. The two highestmass events, while having properties typical for the signal, are still in good agreement with the SM predictions alone. To further illustrate the agreement between the SM background and data, Fig. 3 shows the one-dimensional mass distribution. For reference, the background prediction is 4 events for masses greater than $400 \mathrm{GeV}$, and we see 3 events in the data.
We set limits on the fundamental Planck scale $M_{S}$ via a Bayesian fit to the data with the full signal cross section given by Eq. (1) in the entire $\left(M,\left|\cos \theta^{*}\right|\right)$ plane shown in Fig. 2. The fit parameter $\eta_{G}$ was assumed to have a flat prior distribution. Systematic uncertainties on signal and background were accounted for in the fit and include $K$-factor shape (5\%), the modeling of the $p_{T}$ smearing in the MC (6\%), the dependence on the choice of PDF (5\%), $p_{T}$ dependence of the muon efficiency (5\%), and the MCto-data normalization fit $(1 \%)$. The latter uncertainty accounts for the fact that we used $Z \rightarrow \mu \mu$ events in the signal sample to find the overall normalization for the MC predictions, which reduced significantly the overall uncertainty on the product of the efficiencies and the integrated luminosity. This normalization was done in the $Z$ boson mass peak region from 50 to $120 \mathrm{GeV}$.

The best estimates of the parameter $\eta_{G}$ found in this analysis are
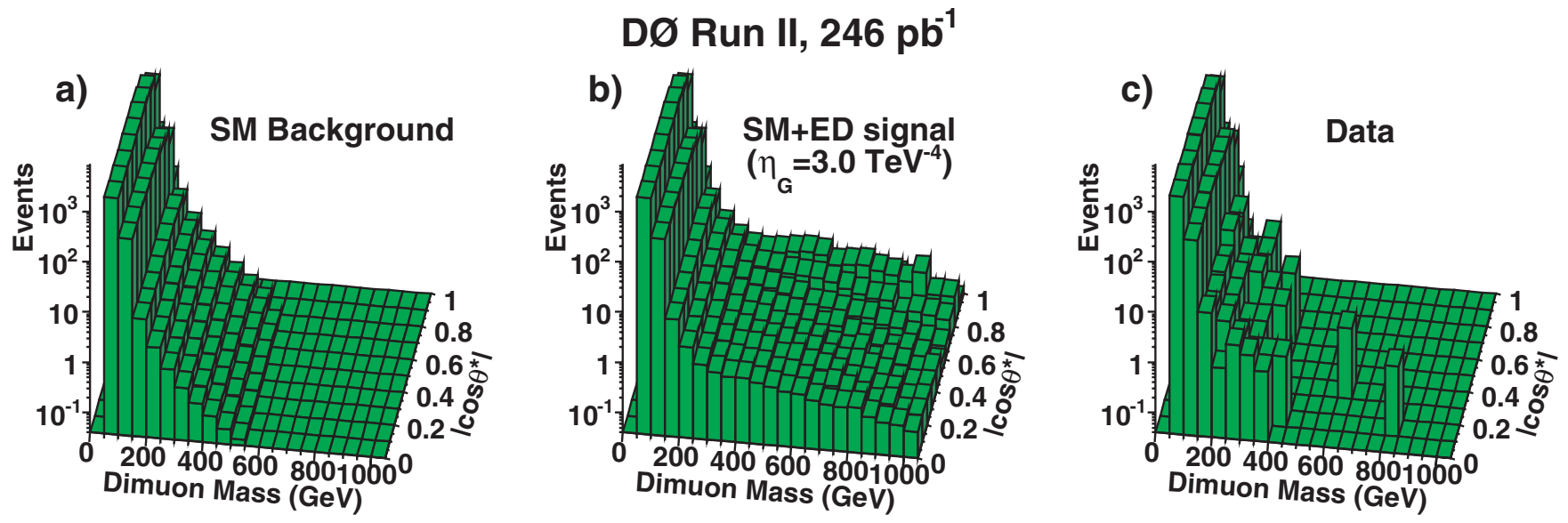

FIG. 2 (color online). Two-dimensional distributions in the dimuon mass vs $\left|\cos \theta^{*}\right|$ for (a) SM background, (b) the sum of the SM and large extra dimensions contributions for $\eta_{G}=3 \mathrm{TeV}^{-4}$, and (c) data. 


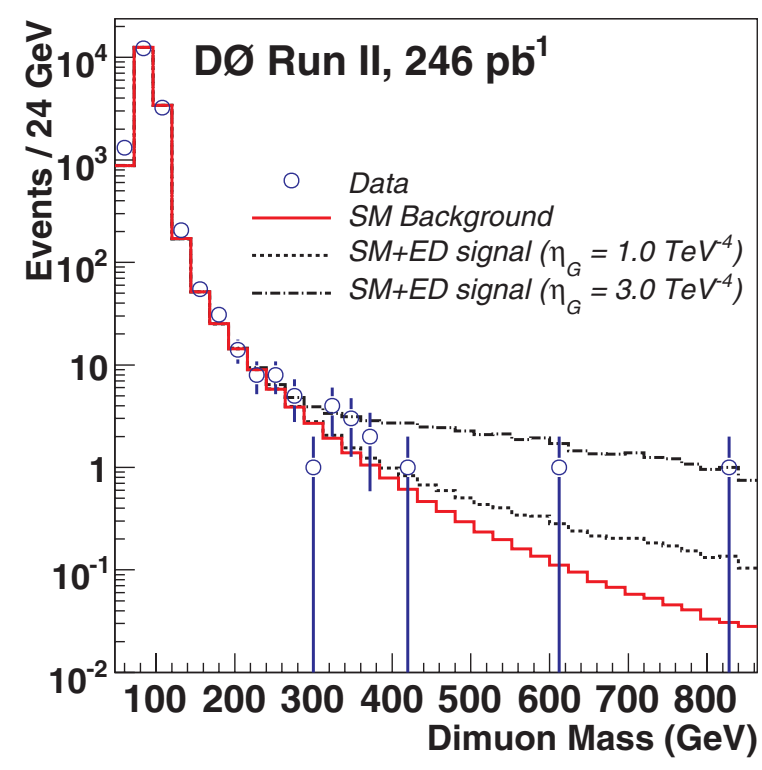

FIG. 3 (color online). Comparison between data and SM background in the dimuon mass $M$, where the effects of extra dimensions are enhanced and shown for $\eta_{G}=1$ and $3 \mathrm{TeV}^{-4}$ (dashed lines).

$$
\begin{gathered}
\eta_{G}=0.00_{-0.00}^{+0.32} \mathrm{TeV}^{-4} \quad\left(\eta_{G} \geq 0\right), \\
\eta_{G}=-0.36 \pm 0.35 \mathrm{TeV}^{-4} \quad\left(\eta_{G} \leq 0\right),
\end{gathered}
$$

which are fully consistent with the SM value of $\eta_{G}=0$. From this the one-sided $95 \%$ C.L. limits on $\eta_{G}$ are

$$
\begin{array}{cc}
\eta_{G}<0.76 \mathrm{TeV}^{-4} & \left(\eta_{G} \geq 0\right), \\
\eta_{G}>-0.84 \mathrm{TeV}^{-4} & \left(\eta_{G} \leq 0\right) .
\end{array}
$$

Our results are in good agreement with the expected sensitivity, as obtained by an ensemble of MC trial experiments $\left(0.76 \mathrm{TeV}^{-4}\right.$ for $\left.\eta_{G}>0\right)$. The use of both the mass and angular variables in the fit allowed for $\approx 7 \%$ improvement in the sensitivity to $\eta_{G}$.

We express these results in terms of limits on the fundamental Planck scale within the three formalisms of Eqs. (2) and (3). In the formalism of Hewett [6], both signs of $\eta_{G}$ are possible and therefore both limits, (8) and (9), are relevant. In the other two formalisms [4,5], $\eta_{G}$ is always positive, and only the first limit is relevant. For the HLZ formalism, the case of $n=2$ is special since $\mathcal{F}$, and therefore $\eta_{G}$, depends on $M^{2}$. To relate $\eta_{G}$ to $M_{S}$ for $n=2$, we used an average $M^{2}$ for the $G_{\mathrm{KK}}$ term at the Tevatron of $(0.64 \mathrm{TeV})^{2}$ [7]. The limits are summarized in Table I.

In summary, we have performed the first search for large extra spatial dimensions in the dimuon channel at hadron colliders by looking for effects of virtual Kaluza-Klein gravitons. We found no evidence for large extra dimensions in this channel with $\approx 250 \mathrm{pb}^{-1}$ of data collected in Run II of the Fermilab Tevatron Collider. We set a $95 \%$
TABLE I. Lower limits at the $95 \%$ C.L. on the fundamental Planck scale, $M_{S}$, in TeV.

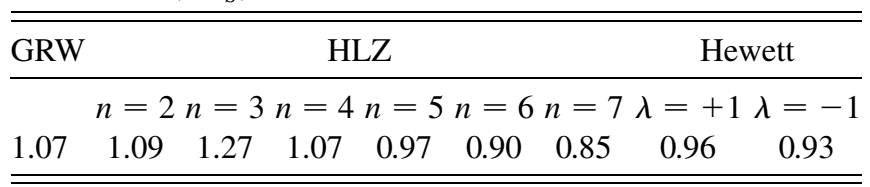

C.L. upper limit of $0.76 \mathrm{TeV}^{-4}$ on the parameter $\eta_{G}$ (for $\eta_{G} \geq 0$ ) that describes the strength of the extra dimensions effects. This result corresponds to limits on the fundamental Planck scale ranging between 0.85 and $1.27 \mathrm{TeV}$ for several formalisms and numbers of large extra dimensions. For comparison in Run I D0 placed a limit on $M_{S}$, in dielectron plus diphoton production, of $1.1 \mathrm{TeV}$ in Hewett's $\lambda=+1$ formalism, while LEP's DELPHI experiment placed a limit on $M_{S}$ in dimuon production of $0.73 \mathrm{TeV}$ in Hewett's $\lambda=+1$ formalism [3]. The limits from this analysis represent the most restrictive achieved in the dimuon channel to date. Results presented here also represent the most precise test of high mass SM Drell-Yan production in this channel at a hadron collider.

We thank the staffs at Fermilab and collaborating institutions, and acknowledge support from the DOE and NSF (USA); CEA and CNRS/IN2P3 (France); FASI, Rosatom, and RFBR (Russia); CAPES, CNPq, FAPERJ, FAPESP, and FUNDUNESP (Brazil); DAE and DST (India); Colciencias (Colombia); CONACyT (Mexico); KRF (Korea); CONICET and UBACyT (Argentina); FOM (The Netherlands); PPARC (United Kingdom); MSMT (Czech Republic); CRC Program, CFI, NSERC, and WestGrid Project (Canada); BMBF and DFG (Germany); SFI (Ireland); Research Corporation, Alexander von Humboldt Foundation, and the Marie Curie Program.

*Visitor from University of Zurich, Zurich, Switzerland.

[1] N. Arkani-Hamed, S. Dimopoulos, and G. Dvali, Phys. Lett. B 429, 263 (1998); Phys. Rev. D 59, 086004 (1999); I. Antoniadis, N. Arkani-Hamed, S. Dimopoulos, and G. Dvali, Phys. Lett. B 436, 257 (1998).

[2] J. L. Hewett and M. Spiropulu, Annu. Rev. Nucl. Part. Sci. 52, 397 (2002); G. Landsberg, in Proceedings of the 10th International Conference on Supersymmetry and Unification of Fundamental Interactions, Hamburg, 2002, edited by P. Nath, P.M. Zerwas, and C. Grosche (Deutsches Electronensynchrotron DESY, Hamburg, 2002), Vol. 1, p. 562; M. Cavaglia, Int. J. Mod. Phys. A 18, 1843 (2003); P. Kanti, Int. J. Mod. Phys. A 19, 4899 (2004).

[3] See, e.g., G. Landsberg, in Proceedings of the 32nd SLAC Summer Institute on Particle Physics, Menlo Park, 2004, eConf C040802, MOT006 (2004).

[4] G. Giudice, R. Rattazzi, and J. Wells, Nucl. Phys. B544, 3 (1999); hep-ph/9811291, revised version 2. (The paper uses the notation $\Lambda_{T}$ for the cutoff scale $M_{S}$ ). 
[5] T. Han, J. D. Lykken, and R.-J. Zhang, Phys. Rev. D 59, 105006 (1999); hep-ph/9811350, revised version 4.

[6] J. L. Hewett, Phys. Rev. Lett. 82, 4765 (1999).

[7] K. Cheung and G. Landsberg, Phys. Rev. D 62, 076003 (2000).

[8] B. Abbott et al. (D0 Collaboration), Phys. Rev. Lett. 86, 1156 (2001).

[9] V. Abazov et al. (D0 Collaboration), physics/0507191 [Nucl. Instrum. Methods Phys. Res., Sect. A (to be published).

[10] S. Abachi et al. (D0 Collaboration), Nucl. Instrum. Methods Phys. Res., Sect. A 338, 185 (1994).

[11] V. Abazov et al. (D0 Collaboration), physics/0503151
[Nucl. Instrum. Methods Phys. Res., Sect. A (to be published)].

[12] We use a right-handed coordinate system with the $z$ axis in the direction of the proton beam and azimuthal angle $\phi$ defined in the plane perpendicular to the beams with $\phi=$ $\pi / 2$ pointing up.

[13] H. L. Lai et al. (CTEQ Collaboration), Phys. Rev. D 55, 1280 (1997).

[14] R. Harnberg, W. L. Van Neerven, and T. Matsura, Nucl. Phys. B359, 343 (1991).

[15] P. Mathews, V. Ravindran, K. Sridhar, and W.L. Van Neerven, Nucl. Phys. B713, 333 (2005). 